\title{
Design and Realization of Intelligent Eyeshade Based on STC89C58RD+
}

\author{
Xin $\mathrm{Li}^{1, \text { a }}$, Wennan Miao ${ }^{1, \mathrm{~b}}$, \\ ${ }^{1}$ Guangzhou College of South China University of \\ Technology, Guangzhou, 510800, China; \\ alixin-820701@163.com, b70324959@qq.com,
}

\author{
Letian Teng ${ }^{2, \mathrm{c}}$ \\ ${ }^{2}$ Guangdong Province 75260 troops, 512100, China; \\ c22923963@qq.com
}

\begin{abstract}
With the accelerated pace of life, busy with work during the day, lack of sleep at night time, people can only catch up on sleep by lunch time, or in the vehicle for a short break, if the external environment is not good will easily affect the quality of sleep. So, we design a intelligent eyeshade, it's main controller is STC89C58RD +, there is an alarm system, a combination of hypnosis and reminder intelligent eyeshade, providing people with a better quality of sleep, is the good assistant.
\end{abstract}

Keywords-lack of sleep, intelligent eyeshade, STC, schedule reminders

\section{INTRODUCTION}

With the acceleration of the rhythm of modern life, nightlife, increased entertainment tool, work pressure gradually increased, the lack of sleep has become a universal phenomenon. Insomnia, dreaminess and other bad sleep toss people increasingly tired irritable, what can be done to improve your sleep? In this paper, the intelligent eyeshade will create a suitable environment for sleep, more suitable for use during the day and outdoor rest at noon, for example, by plane or car; close music player function can not only solve the headset on the ear of bad feeling, but also solve the horn cause resentment of others.

\section{THE PRINCIPLE AND REALIZATION OF THE INTELLIGENT EYESHADE}

The main role of intelligent eyeshade is to block light, eliminate interference, wake and fall asleep easily, so as to create a suitable environment for sleep. The system adopts $89 \mathrm{C} 58 \mathrm{RD}+$ as the main control, low power consumption, high reliability, strong antistatic and anti disturbance, is mainly built by the eyeshade model, MP3 players, micro motors, LED and the corresponding control circuit and so on several major circuits. Overall diagram of system is shown in figure 1

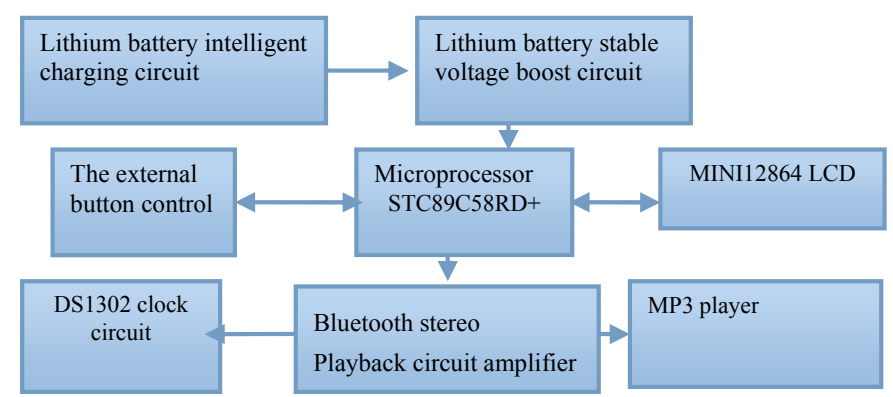

Figure 1 Overall diagram of system

\section{A. Microcontroller STC89C58RD+ selection}

The STC89C58RD+ is a low-power, high performance 8 bit micro controller CMOS, $32 \mathrm{~K}$ system with programmable Flash memory. In a single chip, with 8 bit CPU dexterous and in system programmable Flash, allows the STC89C5X to provide solutions of high flexibility, high effective for many embedded control applications. Has the following functions: standard $32 \mathrm{~K}$ bytes of Flash, 1280 bytes of RAM, 32 bit I/O line, watchdog timer, built-in 16KB EEPROM, MAX810reset circuit, three 16 bit timer / counter, two level interrupt structure of 6 vector, a full duplex serial port ${ }^{[1]}$. In addition, the STC $89 \times 58$ can be reduced to $\mathrm{OHz}$ static logic operation, support 2 software selectable power saving mode. In idle mode, the CPU stops working, RAM, timer / counter, serial port and interrupt to work permit. Power off protection mode, the RAM content being saved, freezes the oscillator, single-chip microcomputer to stop all work, until the next interrupt or hardware reset date. Maximum operating frequency $35 \mathrm{Mhz}$, $6 \mathrm{~T} / 12 \mathrm{~T}$ optional ${ }^{[2]}$. The microprocessor and peripheral circuit schematic diagram is shown in figure 2: 


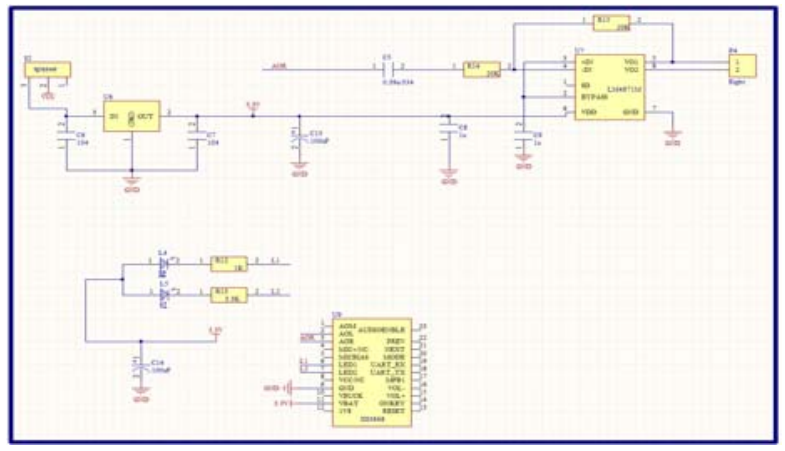

Figure 2 STC89C58RD+ microprocessor and its peripheral circuit principle diagram

\section{B. Rechargeable lithium battery protection chip select}

TP4056 is a complete single lithium ion battery using constant current / constant voltage linear charger. The number of external components of SOP8 package and less at the bottom with a radiating fin makes TP4056 ideal for portable applications. TP4056 can be suitable for USB power supply and power adapters work. Due to the internal PMOSFET structure, plus the anti reverse charging circuit, so do not need external blocking diode. Heat feedback of the charging current can be automatically adjusted, so as to restrict the chip temperature during high power operation or under high temperature conditions. The charge voltage is fixed at $4.2 \mathrm{~V}$, while the charge current can be set externally by a resistor. When the charging current to after reaching the final float voltage setting value $1 / 10$, TP4056 will automatically terminate charging cycle. When the input voltage (AC adapter or USB electric source) is removed, the TP4056 automatically into a low current state, dropping the battery drain current below $2 \mathrm{uA}$. TP4056 in electric source can also be placed in shutdown mode, and will supply current to $55 \mathrm{uA}$. Other features of the TP4056 include a battery temperature detection, undervoltage lockout, automatic recharge and two to indicate charge, the end of the state of the LED pin.

\section{Bluetooth stereo audio chip select}

XS3868 is a highly integrated, low-cost, low power Bluetooth stereo audio module with the Bluetooth 2.0 specification $^{[3]}$. Support A2DP V1.2, AVRCP V1.4 profiles.
EERPOM integration, configurable working mode and parameters; the 6 input keys, including the switch machine key, pair bond, the volume increase, the volume reduction, the last song, the next song; two indicators are used to indicate different working state.

\section{Clock chip select}

DS1302 is a real-time clock circuit, a high performance, low power consumption, with RAM American launched by DALLAS company, it can on the year, month, day, Sunday, when, minutes and seconds for time, has a leap year compensation function, the working voltage is $2.5 \mathrm{~V} \sim 5.5 \mathrm{~V}$. Using three wire interface and CPU communication, and can use a burst mode transmission of multiple byte clock signal or RAM data. DS1302 has an internal $31 \times 8$ for temporary storage of data to the RAM register.

\section{E. Power amplifier chip select}

LM4871 is a bridge type audio power amplifier. The working voltage of $5 \mathrm{~V}$, the largest driving power of $2 \mathrm{~W}(4 \Omega$ BTL load, THD $+\mathrm{N}<10 \%$ ). The application circuit of LM4871 is simple, just a handful of peripheral devices; LM4871 output does not need an external coupling capacitance or the bootstrap capacitor and a buffer network. LM4871 using the SOP package, is especially suitable for the portable system of large volume, small weight; LM4871 can control the entering sleep mode, thereby reducing power consumption; LM4871 internal overheating automatic shutdown protection mechanism.

\section{Design And Realization Of The System SOFTWARE}

The intelligent eyeshade using $89 \mathrm{C} 58 \mathrm{RD}+$ as the main control, mainly composed of DS1302, 18B20, COG and ATJ3310 as the main control MP3 module, Bluetooth, which is safe and reliable and easily fall asleep and wake up in intelligent integrated. Are written to the $\operatorname{cog}$ LCD screen driver $^{[4]}$ and $18 \mathrm{~B} 20$ temperature display $\operatorname{program}^{[5]}$ and DS1302 clock modification function ${ }^{[6]}$, through the main.c file to invoke and set the interface display, menu options, set the date, alarm clock, reminder and the switch of the music and the upper and lower temperature reminding function. The detailed design process of figure 3 , figure 4 flow chart of software design.

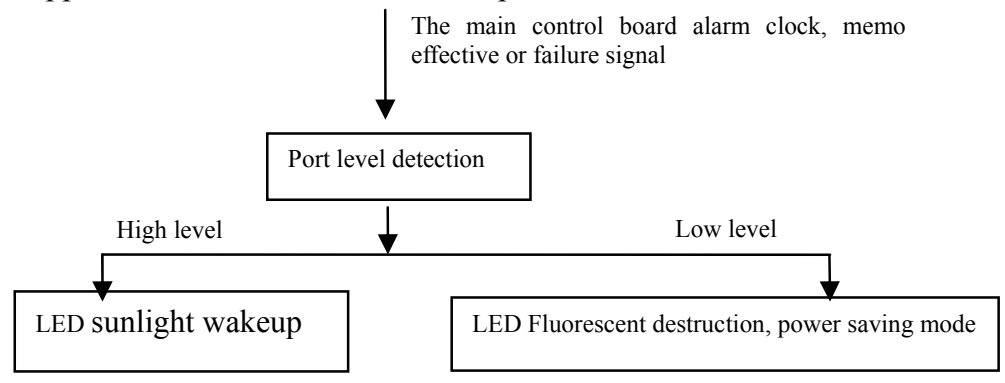

Figure 3. Intelligent eyeshade design flow chart of auxiliary control program 


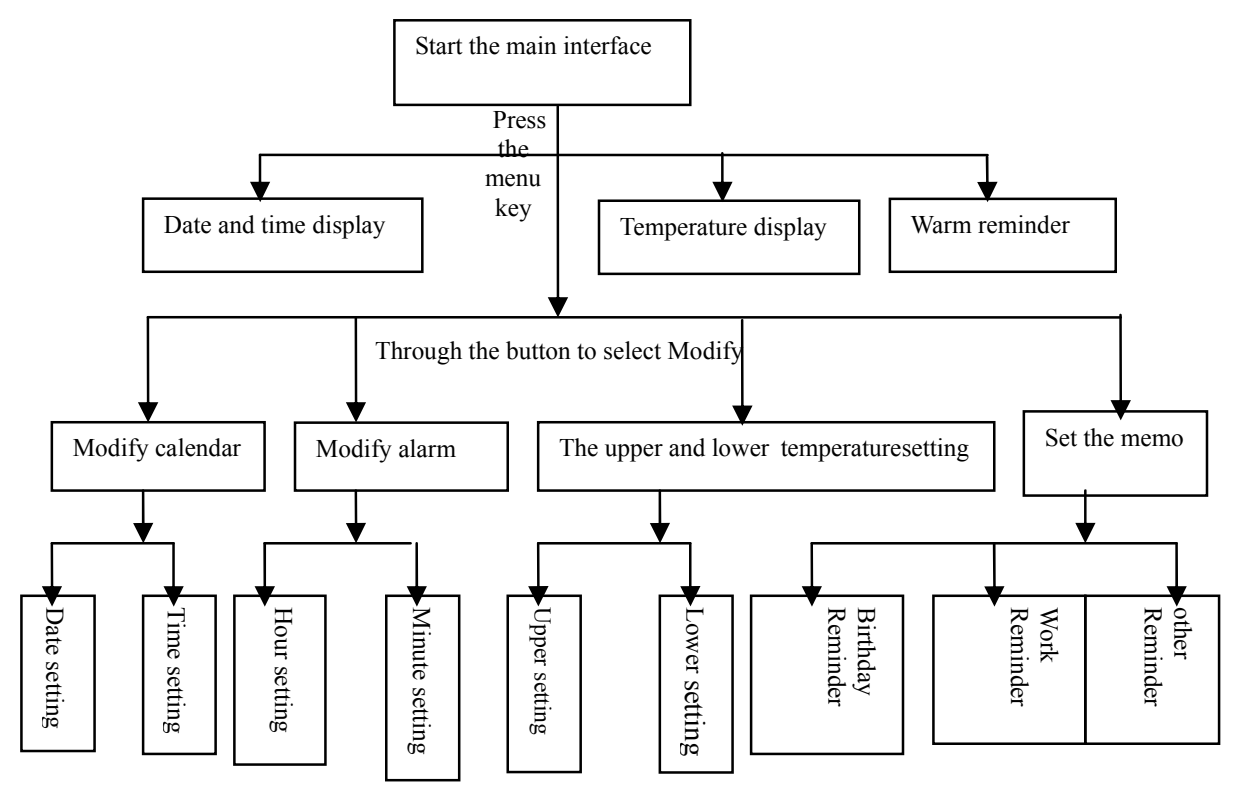

Figure 4. Intelligent eyeshade design flow chart of main control program

\section{CONCLUSION}

Through the test, the eyeshade has basically realized the function of blocking the light, eliminate interference, wake, sleep easy, to create a suitable for sleep environment, and in the choice of the function also has high flexibility, can choose some functions which are combined, for example, can choose only the bluetooth music playing blindfold, also can choose only MP3 playing blindfold, of course, can choose both. People can choose their own scheme according to the specific needs of their own, so it will be more convenient, more humane. It is a good assistant, can help people to improve the quality of sleep, assist in effective rest anywhere daily life, so it has broad market prospects.

\section{REFERENCES}

[1] Tianxiang Guo. new concept 51 microcontroller $\mathrm{C}$ language tutorial: Getting Started, improve, develop and expand the Raiders,Electronic Industry Press,2009.

[2]Shenquan Ma. All model of software system design,Jilin University press, 2013.

[3] Jun Ouyang, Zilong Chen, Ninglin Huang. Bluetooth 4 BLE development completely manual - things development technology combat,Chemical Industry Press, 2013.

[4] Jasmin Blanchette \& Mark Summerfield. C++ GUI Qt4 programming (Second Edition), China material press, 2008.

[5] (Australia) max Asaike. Requirement analysis and system design, Mechanical Industry Press,2009.

[6] Haoqiang Tan.C program design (Fourth Edition), Tsinghua University press, 2010. 